\title{
A SHORT PROOF OF MACDONALD'S CONJECTURE FOR THE ROOT SYSTEMS OF TYPE $A$
}

\author{
JOHN R. STEMBRIDGE
}

(Communicated by Andrew F. Odlyzko)

\begin{abstract}
We give a new proof of I. G. Macdonald's conjecture for the root systems of type $A$ (or equivalently, the equal parameter $q$-Dyson Theorem) that is short, elementary and direct. We also give a short proof of the equal parameter case of a constant term identity due to D. Bressoud and I. Goulden.
\end{abstract}

1. Introduction. In 1982, Macdonald [8] conjectured a series of constant term identities for arbitrary root systems. For the root systems of type $A$, his conjectures were known to be a special case of Andrews' $q$-Dyson conjecture [1], which has been proved by Zeilberger and Bressoud [13]. Furthermore, with the exception of the root system $G_{2}$, the root systems of type $A$ are currently the only cases for which Macdonald's conjecture is known to be true in its full generality. In this paper we present a new proof of Macdonald's conjecture for the root systems of type $A$ that is short (much shorter than that of Zeilberger and Bressoud), elementary, and direct. We remark that very recently, Kadell [7] and Habsieger [4] have independently proved Askey's conjectured $q$-analogue of the Selberg integral. A consequence of this integral is a constant term identity that includes Macdonald's conjecture for the root systems of type $A$ as a special case.

2. The identities and conjectures. We first establish some notation. Let $R$ be a reduced root system of rank $n$ with root lattice $\Lambda$ [6, Chapter III] and exponents $e_{1}, \ldots, e_{n}\left[3\right.$, Chapter 10]. For each $\beta \in \Lambda$, let $x^{\beta}$ denote a formal monomial. More precisely, identify $\left\{x^{\beta}: \beta \in \Lambda\right\}$ as the natural basis of the group $\operatorname{ring} \mathbf{Z}[\Lambda]$. Alternatively, one may choose a base $\Delta$ for $R$ and indeterminates $x^{\alpha}$ for each $\alpha \in \Delta$; the formal monomial $x^{\beta}$ for $\beta \in \Lambda$ may thus be viewed as a Laurent monomial in the variables $x^{\alpha}: \alpha \in \Delta$.

For any formal Laurent series $F$ in the variables $x^{\alpha}: \alpha \in \Delta$, use the notation $\left[x^{\beta}\right] F$ to denote the coefficient of $x^{\beta}$ in $F$.

For any integer $k \geq 0$ (or $k=+\infty$ ), define

$$
(z)_{k}=\prod_{i=1}^{k}\left(1-q^{i-1} z\right)
$$

and for integers $m \geq k \geq 0$, let $\left[\begin{array}{c}m \\ k\end{array}\right]$ denote the $q$-binomial coefficient; i.e.,

$$
\left[\begin{array}{c}
m \\
k
\end{array}\right]=\frac{(q)_{m}}{(q)_{k}(q)_{m-k}} .
$$

Received by the editors October 10, 1986 and, in revised form, February 24, 1987.

1980 Mathematics Subject Classification (1985 Revision). Primary 17B20; Secondary 05A30, 33A30.

Partially supported by N.S.F. Grant number DMS-8603228. 
Macdonald's root system conjecture states the following:

CONJECTURE $2.1[8,(3.1)]$. Let $R^{+}$be a system of positive roots for $R$. We have

$$
\left[x^{0}\right] \prod_{\alpha \in R^{+}}\left(q x^{\alpha}\right)_{k}\left(x^{-\alpha}\right)_{k}=\prod_{i=1}^{n}\left[\begin{array}{c}
k e_{i}+k \\
k
\end{array}\right]
$$

In addition to the root systems of type $A$, Macdonald's conjecture is known to be true in the case $R=G_{2}[\mathbf{1 2}, \mathbf{1 4}]$ and the cases $k=1,2,+\infty$ [8]. Additionally, for the classical root systems $B_{n}, C_{n}, D_{n}$ it is known to be true in the limit $n \rightarrow \infty$ [5] and in the case $q=1$ [8] via Selberg's integral.

As mentioned previously, the cases of type $A$ are special cases of Zeilberger and Bressoud's $q$-Dyson Theorem, which asserts the following:

THEOREM $2.2[\mathbf{1 3}]$. If $a_{1}, \ldots, a_{n}$ are nonnegative integers, then

$$
\left[x^{0}\right] \prod_{1 \leq i<j \leq n}\left(q x_{i} x_{j}^{-1}\right)_{a_{i}}\left(x_{j} x_{i}^{-1}\right)_{a_{j}}=\frac{(q)_{a_{1}+\cdots+a_{n}}}{(q)_{a_{1}} \cdots(q)_{a_{n}}} .
$$

In particular, in the case of equal parameters, we have

$$
\left[x^{0}\right] \prod_{1 \leq i<j \leq n}\left(q x_{i} x_{j}^{-1}\right)_{k}\left(x_{j} x_{i}^{-1}\right)_{k}=\frac{(q)_{k n}}{(q)_{k}^{n}}
$$

which is Macdonald's conjecture for the root system $A_{n-1}$. Our approach to this identity will proceed by first proving a special case of an identity due to Bressoud and Goulden that can be shown to be equivalent to (1). Their identity states the following:

THEOREM $2.3\left[\mathbf{2}\right.$, THEOREM 2.2]. If $a_{1}, \ldots, a_{n}$ are nonnegative integers, then

$$
\left[x^{0}\right] \prod_{1 \leq i<j \leq n}\left(q x_{i} x_{j}^{-1}\right)_{a_{i}-1}\left(x_{j} x_{i}^{-1}\right)_{a_{j}}=\frac{(q)_{a_{1}+\cdots+a_{n}}}{(q)_{a_{1}} \cdots(q)_{a_{n}}} \prod_{i=1}^{n} \frac{1-q^{a_{i}}}{1-q^{a_{i}+\cdots+a_{n}}} .
$$

We will give a proof of the equal parameter case of this identity; namely, the identity

$$
\left[x^{0}\right] \prod_{1 \leq i<j \leq n}\left(q x_{i} x_{j}^{-1}\right)_{k-1}\left(x_{j} x_{i}^{-1}\right)_{k}=\frac{(q)_{k n}}{(q)_{k}^{n}} \cdot \frac{1}{P_{n}\left(q^{k}\right)},
$$

where $P_{n}(z)$ denotes the polynomial

$$
P_{n}(z)=\prod_{i=1}^{n} \frac{1-z^{i}}{1-z}
$$

We mention that this identity is the special case $R=A_{n-1}$ of another root system conjecture due to Macdonald. Although originally stated as an integral over the compact connected Lie group associated with $R$, a straightforward application of 
the Weyl character formula yields an equivalent formulation of the conjecture as a constant term identity:

CONJECTURE $2.4\left[8,\left(3.1^{\prime}\right)\right]$. We have

$$
\left[x^{0}\right] \prod_{\alpha \in R^{+}}\left(q x^{\alpha}\right)_{k-1}\left(x^{-\alpha}\right)_{k}=\prod_{i=1}^{n}\left[\begin{array}{c}
k e_{i}+k-1 \\
k-1
\end{array}\right] .
$$

Macdonald [8] has proved that Conjectures 2.1 and 2.4 are equivalent. Furthermore, Bressoud and Goulden [2] have shown that Theorem 2.3 can be used to prove the $q$-Dyson Theorem, although the proof is as difficult as the proof of the $q$-Dyson Theorem itself. However, when specialized to the case $a_{1}=\cdots=a_{n}=k$, the proof of equivalence is relatively easy. Thus, once the identity (2) is proved, there are at least two ways (Macdonald's or Bressoud and Goulden's) to deduce the original identity (1). So that a complete proof of (1) can be assembled within this paper, we give yet another proof of the equivalence of $(1)$ and (2) in $\S 4$.

3. Proof of the identity (2). For any $\beta=\left(\beta_{1}, \ldots, \beta_{n}\right) \in \mathbf{Z}^{n}$, let $x^{\beta}=$ $x_{1}^{\beta_{1}} \cdots x_{n}^{\beta_{n}}$, and for any formal Laurent series $G\left(x_{1}, \ldots, x_{n}\right)$, let $\left[x^{\beta}\right] G$ denote the coefficient of $x^{\beta}$ in $G$. This conflicts superficially with our previous notion of formal monomials, but it will simplify our subsequent calculations by taking advantage of the natural embedding of the root lattice of $A_{n-1}$ in $\mathbf{Z}^{n}$. A further simplification in our proof of (2) will be in the replacement of the occurrences of $q^{k}$ by another indeterminate $z$. Thus, we consider the formal Laurent series $F_{n}(z, q)=F_{n}\left(z, q ; x_{1}, \ldots, x_{n}\right)$ defined by

$$
F_{n}(z, q)=\prod_{1 \leq i<j \leq n}\left(x_{i}-x_{j}\right) \prod_{1 \leq i \neq j \leq n} \frac{\left(q x_{j} x_{i}^{-1}\right)_{\infty}}{\left(z x_{j} x_{i}^{-1}\right)_{\infty}}
$$

and we prove the following:

THEOREM 3.1. We have

$$
\left[x^{\delta}\right] F_{n}(z, q)=\frac{(q z)_{\infty}^{n}}{(q)_{\infty}^{n-1}\left(q z^{n}\right)_{\infty} P_{n}(z)}
$$

where $\delta=(n-1, n-2, \ldots, 1,0)$.

The identity (2) can be obtained as an immediate corollary via the specialization $z=q^{k}$ and the observation

$$
\prod_{i<j}\left(x_{i}-x_{j}\right)=x^{\delta} \prod_{i<j}\left(1-x_{j} x_{i}^{-1}\right) .
$$

Theorem 3.1 should only be considered a superficial generalization of (2) because the following lemma shows that, conversely, (2) implies Theorem 3.1.

LEMMA 3.2. Let $F(z, q)$ and $G(z, q)$ be formal power series. If $F\left(q^{k}, q\right)=$ $G\left(q^{k}, q\right)$ for infinitely many integers $k \geq 0$, then $F=G$.

ProOF. Assume that $F(z, q)$ and $G(z, q)$ provide a counterexample. The series $H=F-G$ is nonzero, but $H\left(q^{k}, q\right)=0$ for infinitely many $k \geq 0$. Certainly $H$ has an expansion of the form $H(z, q)=z^{r} H_{r}(q)+z^{r+1} H_{r+1}(q)+\cdots$ where $H_{r} \neq 0$. If 
$H\left(q^{k}, q\right)=0$, it follows that $H_{r}(q)$ can only have terms of order $q^{k}$ or higher. Since the possibilities for $k$ are unbounded, we must have $H_{r}=0$, a contradiction.

A crucial property of the series $F_{n}(z, q)$ that makes evaluation of $\left[x^{\delta}\right] F_{n}(z, q)$ easier than the evaluation of the constant term in Theorem 2.3 is the fact that $F_{n}(z, q)$ is antisymmetric under permutations of the variables $x_{1}, \ldots, x_{n}$; i.e., $w F_{n}(z, q)=(\operatorname{sgn} w) F_{n}(z, q)$ for every $w \in S_{n}$.

For any $\beta \in \mathbf{Z}^{n}$, let $a_{\beta}\left(x_{1}, \ldots, x_{n}\right)$ denote the monomial antisymmetric function defined by

$$
a_{\beta}=\operatorname{det}\left[x_{i}^{\beta_{j}}\right]=\sum_{w \in S_{n}}(\operatorname{sgn} w) x^{w \beta} .
$$

Let $\Gamma_{n}=\left\{\beta \in \mathbf{Z}^{n}: \beta_{1} \geq \cdots \geq \beta_{n}\right\}$, and note that $\left\{a_{\beta+\delta}: \beta \in \Gamma_{n}\right\}$ forms a $\mathbf{Z}$-basis for the antisymmetric polynomials in $\mathbf{Z}\left[x_{1}^{ \pm 1}, \ldots, x_{n}^{ \pm 1}\right]$. Since $F_{n}(z, q)$ is antisymmetric, it follows that there are unique formal power series $c_{n}^{\beta}=c_{n}^{\beta}(z, q)$ for each $\beta \in \Gamma_{n}$ such that

$$
F_{n}(z, q)=\sum_{\beta \in \Gamma_{n}} c_{n}^{\beta}(z, q) a_{\beta+\delta}
$$

namely, $c_{n}^{\beta}(z, q)=\left[x^{\beta+\delta}\right] F_{n}(z, q)$.

We remark that the series $c_{n}^{\beta}(z, q)$ have been studied in [11], where, for example, an explicit formula for $c_{n}^{\beta}(z, q)$ is given for those $\beta \in \Gamma_{n}$ with $\beta_{n}=-1$. One reason for the interest in these series is their connection to the representation theory of $S L_{n}$. For example, the coefficient of $z^{l}$ in $(1-z)^{-n+1} c_{n}^{\beta}(z, 0)$ is the multiplicity of the irreducible $S L_{n}$-module corresponding to $\beta$ in the $l$ th symmetric power $\operatorname{Sym}^{l}\left(s l_{n}\right)$, and the coefficient of $q^{l}$ in $(q)_{k}^{n-1} c_{n}^{\beta}\left(q^{k+1}, q\right)$ is the Euler characteristic of the $\beta$ th isotypic component of the $k$ th Macdonald complex of $s l_{n}$. See [11] for details.

In the following, it will be convenient to regard $\left[x^{\beta}\right] G$ as a bilinear operation. More precisely, define $[F] G$ for polynomials $F \in \mathbf{Z}\left[x_{1}^{ \pm 1}, \ldots, x_{n}^{ \pm 1}\right]$ and formal Laurent series $G \in \mathbf{Z}\left[\left[x_{1}^{ \pm 1}, \ldots, x_{n}^{ \pm 1}\right]\right]$ (or any extension of the coefficient ring, such as $\mathbf{Z}[[z, q]])$ via

$$
\left[\sum c_{\beta} x^{\beta}\right] G=\sum c_{\beta}\left[x^{\beta}\right] G
$$

Notice that multiplication by $H \in \mathbf{Z}\left[x_{1}^{ \pm 1}, \ldots, x_{n}^{ \pm 1}\right]$ is adjoint to multiplication by $H\left(x^{-1}\right)=H\left(x_{1}^{-1}, \ldots, x_{n}^{-1}\right)$, i.e.,

$$
[F H] G(x)=[F] G(x) H\left(x^{-1}\right) .
$$

LEMMA 3.3 (CF. [11, LEMMA 5.4]). For any $\beta \in \Gamma_{n}$ we have

$$
q^{\beta_{n}}\left[x^{\beta+\delta} \prod_{i=1}^{n-1}\left(1-z \frac{x_{n}}{x_{i}}\right)\right] F_{n}(z, q)=z^{n-1}\left[x^{\beta+\delta} \prod_{i=1}^{n-1}\left(1-z^{-1} \frac{x_{n}}{x_{i}}\right)\right] F_{n}(z, q) \text {. }
$$

ProOF. We have

$$
\begin{aligned}
& q^{\beta_{n}}\left[x^{\beta+\delta} \prod_{i=1}^{n-1}\left(1-z \frac{x_{n}}{x_{i}}\right)\right] F_{n}(z, q) \\
& \quad=\left[x^{\beta+\delta}\right] F_{n}\left(z, q ; x_{n} \rightarrow q x_{n}\right) \prod_{i=1}^{n-1}\left(1-\frac{z x_{i}}{q x_{n}}\right)
\end{aligned}
$$




$$
z^{n-1}\left[x^{\beta+\delta} \prod_{i=1}^{n-1}\left(1-z^{-1} \frac{x_{n}}{x_{i}}\right)\right] F_{n}(z, q)=\left[x^{\beta+\delta}\right] F_{n}(z, q) \prod_{i=1}^{n-1}\left(z-\frac{x_{i}}{x_{n}}\right) .
$$

The right sides of these identities agree, due to the fact that

$$
\frac{F_{n}\left(z, q ; x_{n} \rightarrow q x_{n}\right)}{F_{n}(z, q)}=\prod_{i=1}^{n-1} \frac{z-x_{i} x_{n}^{-1}}{1-z q^{-1} x_{i} x_{n}^{-1}},
$$

which is a straightforward algebraic calculation.

Since the series $F_{n}(z, q)$ is antisymmetric, it follows that $\left[x^{\gamma}\right] F_{n}(z, q)$ vanishes unless the terms of $\gamma \in \mathbf{Z}^{n}$ are distinct. If they are distinct, then there is a unique $w \in S_{n}$ and a unique $\beta \in \Gamma_{n}$ such that $\gamma=w(\beta+\delta)$, and we have $\left[x^{\gamma}\right] F_{n}(z, q)=(\operatorname{sgn} w)\left[x^{\beta+\delta}\right] F_{n}(z, q)=(\operatorname{sgn} w) c_{n}^{\beta}(z, q)$. Thus, Lemma 3.3 gives a collection of homogeneous linear equations satisfied by the series $c_{n}^{\beta}(z, q)$. It can be shown [11, Theorem 5.1] that these linear equations can be ordered so that they are in triangular form and given an explicit combinatorial description. For our purposes, it will suffice to restrict our attention to a certain subcollection of the equations corresponding to those $\beta \in \Gamma_{n}$ of the form

$$
\beta=\beta(r, s):=((r-1)(n-1)+s,-\underbrace{(r-1), \ldots,-(r-1)}_{n-1-s},-\underbrace{r, \ldots,-r}_{s}),
$$

where $r \geq 1,0 \leq s \leq n-1$. Notice that $\beta(r, 0)=\beta(r-1, n-1)$.

The equation obtained by choosing $\beta=\beta(r, s)$ in Lemma 3.3 has an especially simple form, as the following result indicates. Although it follows directly from [11, Theorem 5.1], we include a proof for the sake of completeness.

LEMMA 3.4. Let $y$ be an indeterminate, and let $\beta=\beta(r, s)$ where $r \geq 1$ and $1 \leq s \leq n-1$. We have

$$
\left[x^{\beta+\delta} \prod_{i=1}^{n-1}\left(1-y \frac{x_{n}}{x_{i}}\right)\right] F_{n}(z, q)=\left(\frac{1-y^{s}}{1-y}\right) c_{n}^{\beta(r, s)}-\left(\frac{y^{s}-y^{n}}{1-y}\right) c_{n}^{\beta(r, s-1)} .
$$

PROOF. Notice that

$$
\prod_{i=1}^{n-1}\left(1-y \frac{x_{n}}{x_{i}}\right)=\sum_{T}\left(-y x_{n}\right)^{|T|} x^{-T}
$$

summed over all $T \subseteq\{1, \ldots, n-1\}$, where $x^{-T}$ is used as an abbreviation for $\prod_{i \in T} x_{i}^{-1}$. Since

$$
\beta+\delta=(r(n-1)+s, \underbrace{n-r-1, \ldots, s-r+1}_{n-1-s}, \underbrace{s-r-1, \ldots,-r}_{s}),
$$

where the braces indicate consecutive integers, it is not hard to see that for each $t(0 \leq t \leq n-1)$ there is a unique $t$-set $T \subseteq\{1, \ldots, n-1\}$ such that the monomial $x^{\beta+} x_{n}^{t} x^{-T}$ has distinct exponents; namely,

$$
T= \begin{cases}\{n-t, \ldots, n-1\} & \text { if } 0 \leq t \leq s-1 \\ \{1, n-t+1, \ldots, n-1\} & \text { if } s \leq t \leq n-1\end{cases}
$$


Furthermore, observe that for these choices of $T$, we have

$$
x^{\beta+\delta} x_{n}^{t} x^{-T}= \begin{cases}x^{w_{t}(\beta(r, s)+\delta)} & \text { if } \quad 0 \leq t \leq s-1 \\ x^{w_{t}(\beta(r, s-1)+\delta)} & \text { if } \quad s \leq t \leq n-1\end{cases}
$$

where $w_{t}$ is a $(t+1)$-cycle if $0 \leq t \leq s-1$ and $w_{t}$ is a $t$-cycle if $s \leq t \leq n-1$. Thus, we have shown

$$
\begin{aligned}
{\left[x^{\beta+\delta} \prod_{i=1}^{n-1}\left(1-y \frac{x_{n}}{x_{i}}\right)\right] F_{n}(z, q)=} & {\left[\sum_{t=0}^{s-1}(-1)^{t} y^{t} x^{w_{t}(\beta(r, s)+\delta)}\right] F_{n}(z, q) } \\
& +\left[\sum_{t=s}^{n-1}(-1)^{t} y^{t} x^{w_{t}(\beta(r, s-1)+\delta)}\right] F_{n}(z, q)
\end{aligned}
$$

which yields the desired result, since $\left[x^{w(\gamma+\delta)}\right] F_{n}(z, q)=(\operatorname{sgn} w) c_{n}^{\gamma}(z, q)$ for all $w \in S_{n}$ and $\gamma \in \Gamma_{n}$.

We are now ready to give the

PROOF OF THEOREM 3.1. Apply Lemma 3.4 to both halves of the identity in Lemma 3.3; the left side with $y=z$, the right side with $y=z^{-1}$. The result is a linear relation between $c_{n}^{\beta(r, s)}$ and $c_{n}^{\beta(r, s-1)}$; namely,

$$
c_{n}^{\beta(r, s)}(z, q)=\frac{\left(1-z^{n-s}\right)\left(z^{s}-q^{r}\right)}{\left(1-z^{s}\right)\left(1-q^{r} z^{n-s}\right)} c_{n}^{\beta(r, s-1)}(z, q) .
$$

If we iterate this recursion $n-1$ times and recognize the fact that $\beta(r+1,0)=$ $\beta(r, n-1)$, we discover

$$
c_{n}^{\beta(r+1,0)}=c_{n}^{\beta(r, 0)} \prod_{s=1}^{n-1} \frac{z^{s}-q^{r}}{1-q^{r} z^{s}},
$$

and hence,

$$
c_{n}^{\beta(k, 0)}=c_{n}^{0} \prod_{r=1}^{k-1} \prod_{s=1}^{n-1} \frac{z^{s}-q^{r}}{1-q^{r} s^{s}}
$$

In particular, we have

(5) $c_{n}^{\beta(k, 0)}\left(q^{k}, q\right)=(-1)^{(n-1)(k-1)} q^{(n-1)\left(\begin{array}{c}k \\ 2\end{array}\right)} \cdot \frac{(q)_{k}}{\left(q^{k(n-1)+1}\right)_{k}} \cdot \frac{\left(1-q^{k n}\right)}{1-q^{k}} c_{n}^{0}\left(q^{k}, q\right)$. (6)

On the other hand, from the definition of the series $c_{n}^{\beta}(z, q)$, we have

$$
c_{n}^{\beta(k, 0)}\left(q^{k}, q\right)=\left[x_{1}^{(k-1)(n-1)} x_{2}^{-(k-1)} \cdots x_{n}^{-(k-1)}\right] \prod_{1 \leq i<j \leq n}\left(x_{j} x_{i}^{-1}\right)_{k}\left(q x_{i} x_{j}^{-1}\right)_{k-1} .
$$

In order to obtain a term from the polynomial

$$
\prod_{i<j}\left(x_{j} x_{i}^{-1}\right)_{k}\left(q x_{i} x_{j}^{-1}\right)_{k-1}
$$

whose $x_{1}$-degree is $(k-1)(n-1)$, we are forced to choose $-q^{l} x_{1} x_{j}^{-1}$ from every binomial of the form $\left(1-q^{l} x_{1} x_{j}^{-1}\right)(1 \leq l \leq k-1,2 \leq j \leq n)$, and we are forced to 
choose 1 from every binomial of the form $\left(1-q^{l} x_{j} x_{1}^{-1}\right)(0 \leq l \leq k-1,2 \leq j \leq n)$. Thus, the variable $x_{1}$ may be eliminated from (6), yielding

(7)

$$
\begin{aligned}
c_{n}^{\beta(k, 0)}\left(q^{k}, q\right) & =(-1)^{(n-1)(k-1)} q^{(n-1)\left(\begin{array}{c}
k \\
2
\end{array}\right)\left[x_{2}^{0} \cdots x_{n}^{0}\right]} \prod_{2 \leq i<j \leq n}\left(x_{j} x_{i}^{-1}\right)_{k}\left(q x_{i} x_{j}^{-1}\right)_{k-1} \\
& =(-1)^{(n-1)(k-1)} q^{(n-1)}\left(\begin{array}{l}
k \\
2
\end{array}\right) c_{n-1}^{0}\left(q^{k}, q\right) .
\end{aligned}
$$

In view of (j), we may therefore deduce

$$
c_{n}^{0}\left(q^{k}, q\right)=\frac{\left(q^{k(n-1)+1}\right)_{k}}{(q)_{k}} \cdot \frac{1-q^{k}}{1-q^{k n}} c_{n-1}^{0}\left(q^{k}, q\right) .
$$

Since $c_{1}^{0}\left(q^{k}, q\right)=1$, it follows that

$$
c_{n}^{0}\left(q^{k}, q\right)=\frac{(q)_{k n}}{(q)_{k}^{n}} \cdot \frac{1}{P_{n}\left(q^{k}\right)},
$$

which is the identity (2). Thus, we have shown that the series

$$
c_{n}^{0}(z, q) \text { and } \frac{(q z)_{\infty}^{n}}{(q)_{\infty}^{n-1}\left(q z^{n}\right)_{\infty} P_{n}(z)}
$$

agree for infinitely many of the special cases $z=q^{k}$. Apply Lemma 3.2.

We remark that a consequence of this proof is the fact that all of the series $c_{n}^{\beta(r, s)}(z, q)$ have simple explicit formulas (viz., (3), (4)).

4. Equivalence of the identities (1) and (2). Finally, we complete our proof of Macdonald's conjecture for the root systems of type $A$ by proving

THEOREM 4.1. We have

$$
\left[x^{0}\right] \prod_{1 \leq i<j \leq n} \frac{\left(x_{j} x_{i}^{-1}\right)_{\infty}\left(q x_{i} x_{j}^{-1}\right)_{\infty}}{\left(z x_{j} x_{i}^{-1}\right)_{\infty}\left(q z x_{i} x_{j}^{-1}\right)_{\infty}}=\frac{(q z)_{\infty}^{n}}{(q)_{\infty}^{n-1}\left(q z^{n}\right)_{\infty}} .
$$

The identity (1) can be obtained via the specialization $z=q^{k}$.

PROOF. Notice that

$$
\prod_{i<j} \frac{\left(x_{j} x_{i}^{-1}\right)_{\infty}\left(q x_{i} x_{j}^{-1}\right)_{\infty}}{\left(z x_{j} x_{i}^{-1}\right)_{\infty}\left(q z x_{i} x_{j}^{-1}\right)_{\infty}}=x^{-\delta} F_{n}(z, q) \prod_{i<j}\left(1-z x_{i} x_{j}^{-1}\right) .
$$

Thus, we seek to prove that

$$
\left[x^{0}\right] x^{-\delta} F_{n}(z, q) \prod_{i<j}\left(1-z x_{i} x_{j}^{-1}\right)=\frac{(q z)_{\infty}^{n}}{(q)_{\infty}^{n-1}\left(q z^{n}\right)_{\infty}} .
$$

By Theorem 3.1, this is equivalent to proving

$$
\left[x^{\delta} \prod_{i<j}\left(1-z x_{j} x_{i}^{-1}\right)\right] F_{n}(z, q)=P_{n}(z)\left[x^{\delta}\right] F_{n}(z, q) .
$$

Let $\varphi$ denote the linear transformation on the vector space of antisymmetric polynomials in $\mathbf{Z}\left[x_{1}^{ \pm 1}, \ldots, x_{n}^{ \pm 1}\right]$ defined by the condition

$$
a_{\alpha} \underset{\varphi}{\mapsto}\left[x^{\alpha}\right] F_{n}(z, q)
$$


for all $\alpha \in \mathbf{Z}^{n}$. This is well defined since $F_{n}(z, q)$ is antisymmetric. By linearity, it follows that for any $G \in \mathbf{Z}\left[x_{1}^{ \pm 1}, \ldots, x_{n}^{ \pm 1}\right]$, we have

$$
\sum_{w \in S_{n}}(\operatorname{sgn} w) w G(x) \underset{\varphi}{\mapsto}[G] F_{n}(z, q)
$$

In particular, from the identity [10, III, (1.4)]

$$
\sum_{w \in S_{n}} w\left(\prod_{i<j} \frac{x_{i}-z x_{j}}{x_{i}-x_{j}}\right)=P_{n}(z)
$$

we have

$$
\sum_{w \in S_{n}}(\operatorname{sgn} w) w\left(\prod_{i<j}\left(x_{i}-z x_{j}\right)\right)=P_{n}(z) \prod_{i<j}\left(x_{i}-x_{j}\right)
$$

so that

$$
P_{n}(z) \prod_{i<j}\left(x_{i}-x_{j}\right) \underset{\varphi}{\mapsto}\left[x^{\delta} \prod_{i<j}\left(1-z x_{j} x_{i}^{-1}\right)\right] F_{n}(z, q)
$$

On the other hand, since

$$
\prod_{i<j}\left(x_{i}-x_{j}\right)=\sum_{w \in S_{n}}(\operatorname{sgn} w) x^{w \delta}
$$

we have

$$
P_{n}(z) \prod_{i<j}\left(x_{i}-x_{j}\right) \underset{\varphi}{\mapsto} P_{n}(z)\left[x^{\delta}\right] F_{n}(z, q) .
$$

In view of (9), the theorem now follows.

We remark that the series $F_{n}(z, q)$ played no essential role in this argument; we have proved that

$$
\left[\prod_{i<j}\left(x_{i}-z x_{j}\right)\right] F=P_{n}(z)\left[x^{\delta}\right] F
$$

for any antisymmetric formal Laurent series $F$.

5. The other root systems. It is natural to hope that the techniques we have presented can be used to prove Macdonald's conjectures for the other root systems; indeed, it is tantalizing to realize that nearly every aspect of the proof easily generalizes to arbitrary root systems.

The role of the series $F_{n}(z, q)$ is played by

$$
F_{R}(z, q)=\prod_{\alpha \in R^{+}}\left(x^{\alpha / 2}-x^{-\alpha / 2}\right) \prod_{\alpha \in R} \frac{\left(q x^{\alpha}\right)_{\infty}}{\left(z x^{\alpha}\right)_{\infty}}
$$

and the corresponding analogue of Theorem 3.1 is the conjecture

$$
\left[x^{\delta}\right] F_{R}(z, q)=\prod_{i=1}^{n} \frac{(z)_{\infty}\left(q z^{e_{i}}\right)_{\infty}}{(q)_{\infty}\left(z^{e_{i}+1}\right)_{\infty}}
$$


where $\delta=\frac{1}{2} \sum_{\alpha \in R^{+}} \alpha$. By Lemma 3.2, this is equivalent to Macdonald's conjecture 2.4.

It is not hard to find functional equations involving $F_{R}(z, q)$ which yield analogues of Lemma 3.3. As with the root systems of type $A$, they give linear equations satisfied by the coefficient series $\left[x^{\alpha+\delta}\right] F_{R}(z, q)$ where $\alpha \in \Lambda$ ranges over dominant weights, and these equations may be ordered so that the resulting system is triangular. It is also not hard to find generalizations of (7); i.e., dominant weights $\beta$ for which $\left[x^{\beta+\delta}\right] F_{R}(z, q)$ can be expressed in terms of $\left[x^{\delta}\right] F_{R^{\prime}}(z, q)$, where $R^{\prime}$ denotes a root system of rank $n-1$. However, it is Lemma 3.4, which shows that some of the linear equations for the coefficients have an especially simple form, that is most resistant to generalization. If the techniques we have presented here are to succeed in general, some way to interpolate between the coefficient $\left[x^{\delta}\right] F_{R}(z, q)$ and the coefficients $\left[x^{\beta+\delta}\right] F_{R}(z, q)$ related to $\left[x^{\delta}\right] F_{R^{\prime}}(z, q)$ must be found.

Finally, we remark that the proof of Theorem 4.1 easily generalizes to other root systems. The identity (10) is replaced with the Weyl denominator formula, and the identity (8) is merely a special case of a beautiful identity due to Macdonald [9, (2.16)]:

$$
\sum_{w \in W} w\left(\prod_{\alpha \in R^{+}} \frac{1-z x^{-\alpha}}{1-x^{-\alpha}}\right)=\prod_{i=1}^{n} \frac{1-z^{e_{i}+1}}{1-z},
$$

where $W$ is the Weyl group of $R$. The resulting $z$-analogue of Conjecture 2.1 and $R$-analogue of Theorem 4.1 is the conjecture

$$
\left[x^{0}\right] \prod_{\alpha \in R^{+}} \frac{\left(x^{-\alpha}\right)_{\infty}\left(q x^{\alpha}\right)_{\infty}}{\left(z x^{-\alpha}\right)_{\infty}\left(q z x^{\alpha}\right)_{\infty}}=\prod_{i=1}^{n} \frac{(q z)_{\infty}\left(q z^{e_{i}}\right)_{\infty}}{(q)_{\infty}\left(q z^{e_{i}+1}\right)_{\infty}}
$$

\section{REFERENCES}

1. G. E. Andrews, Problems and prospects for basic hypergeometric functions, Theory and Application of Special Functions (R. Askey, ed.), Academic Press, New York, 1975, pp. 191-224.

2. D. M. Bressoud and I. P. Goulden, Constant term identities extending the q-Dyson theorem, Trans. Amer. Math. Soc. 291 (1985), 203-228.

3. R. W. Carter, Simple groups of Lie type, Wiley, London and New York, 1972.

4. L. Habsieger, Une q-intégrale de Selberg Askey, SIAM J. Math. Anal. (to appear); summarized in C. R. Acad. Sci. Paris (I) 302 (1986), 615-617.

5. P. Hanlon, The proof of a limiting case of Macdonald's root system conjectures, Proc. London Math. Soc. (3) 49 (1984), 170-182.

6. J. E. Humphreys, Introduction to Lie algebras and representation theory, Springer-Verlag, New York, Heidelberg and Berlin, 1972.

7. K. W. J. Kadell, A proof of Askey's conjectured $q$-analog of Selberg's integral and a conjecture of Morris, SIAM J. Math. Anal. (to appear).

8. I. G. Macdonald, Some conjectures for root systems, SIAM J. Math. Anal. 13 (1982), 988-1007.

9. __ The Poincaré series of a Coxeter group, Math. Ann. 199 (1972), 161-174.

10. __ Symmetric functions and Hall polynomials, Oxford, 1979.

11. J. R. Stembridge, First layer formulas for characters of $S L(n, \mathbf{C})$, Trans. Amer. Math. Soc. 299 (1987), 319350. 
12. D. Zeilberger, $A$ proof of the $G_{2}$ case of Macdonald's root system-Dyson conjecture, preprint.

13. D. Zeilberger and D. Bressoud, A proof of Andrews' q-Dyson conjecture, Discrete Math. 54 (1985), 201-224.

14. L. Habsieger, La q-conjecture de Macdonald-Morris pour $G_{2}$, C. R. Acad. Sci. Paris (I) 303 (1986), 211-213.

Department of Mathematics, University of California, Los Angeles, CaliFORNIA 90024 